\title{
Polymorphisms in signal transducer and activator of transcription 3 and lung function in asthma
}

\author{
Augusto A Litonjua*1,2,4, Kelan G Tantisira1,3,4, Stephen Lake ${ }^{1,4}$, \\ Ross Lazarus ${ }^{1,4}$, Brent G Richter ${ }^{1}$, Stacey Gabriel ${ }^{5}$, Eric S Silverman ${ }^{3,4}$ and \\ Scott T Weiss ${ }^{1,4}$
}

\begin{abstract}
Address: ${ }^{1}$ Channing Laboratory, Department of Medicine, Brigham and Women's Hospital, 181 Longwood Avenue, Boston, MA 02115 USA, 2Division of Pulmonary and Critical Care Medicine, Beth Israel Deaconess Medical Center, 330 Brookline Avenue, Boston, MA 02215 USA, ${ }^{3}$ Pulmonary Division, Brigham and Women's Hospital, 75 Francis Street, Boston, MA 02115 USA, ${ }^{4}$ Harvard Medical School, 25 Shattuck Street, Boston, MA 02115 USA and ${ }^{5}$ Whitehead Institute, Massachusetts Institute of Technology, Nine Cambridge Center, Cambridge, MA 02142 USA

Email: Augusto A Litonjua* - augusto.litonjua@channing.harvard.edu; Kelan G Tantisira - kelan.tantisira@channing.harvard.edu; Stephen Lake - stephen.lake@channing.harvard.edu; Ross Lazarus - ross.lazarus@channing.harvard.edu; Brent G Richter - brent.richter@channing.harvard.edu; Stacey Gabriel - stacy@genome.wi.mit.edu;

Eric S Silverman - essilverma@bics.bwh.harvard.edu; Scott T Weiss - scott.weiss@channing.harvard.edu

* Corresponding author
\end{abstract}

Published: 03 June 2005

Respiratory Research 2005, 6:52 doi:10.1 186/1465-992I-6-52

This article is available from: http://respiratory-research.com/content/6/1/52

(c) 2005 Litonjua et al; licensee BioMed Central Ltd.

This is an Open Access article distributed under the terms of the Creative Commons Attribution License (http://creativecommons.org/licenses/by/2.0), which permits unrestricted use, distribution, and reproduction in any medium, provided the original work is properly cited.
Received: 01 December 2004

Accepted: 03 June 2005

\begin{abstract}
Background: Identifying genetic determinants for lung function is important in providing insight into the pathophysiology of asthma. Signal transducer and activator of transcription 3 is a transcription factor latent in the cytoplasm; the gene (STAT3) is activated by a wide range of cytokines, and may play a role in lung development and asthma pathogenesis.
\end{abstract}

Methods: We genotyped six single nucleotide polymorphisms (SNPs) in the STAT3 gene in a cohort of 401 Caucasian adult asthmatics. The associations between each SNP and forced expiratory volume in I second $\left(\mathrm{FEV}_{1}\right)$, as a percent of predicted, at the baseline exam were tested using multiple linear regression models. Longitudinal analyses involving repeated measures of FEV were conducted with mixed linear models. Haplotype analyses were conducted using imputed haplotypes. We completed a second association study by genotyping the same six polymorphisms in a cohort of 652 Caucasian children with asthma.

Results: We found that three polymorphisms were significantly associated with baseline $\mathrm{FEV}_{\mathrm{l}}$ : homozygotes for the minor alleles of each polymorphism had lower FEV, than homozygotes for the major alleles. Moreover, these associations persisted when we performed an analysis on repeated measures of FEV , over 8 weeks. A haplotypic analysis based on the six polymorphisms

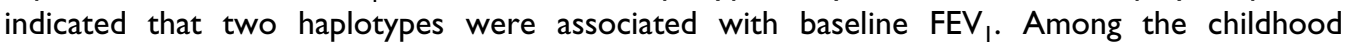
asthmatics, one polymorphism was associated with both baseline FEV , and the repeated measures of $\mathrm{FEV}$, over 4 years.

Conclusion: Our results indicate that genetic variants in STAT3, independent of asthma treatment, are determinants of $\mathrm{FEV}_{1}$ in both adults and children with asthma, and suggest that STAT3 may participate in inflammatory pathways that have an impact on level of lung function. 


\section{Background}

It is recognized that genetic factors influence lung function $[1,2]$. The identification of genetic variants that determine either lung function development or decline is particularly important for diseases in which low lung function is a feature, such as chronic obstructive pulmonary disease and asthma, since this provides insight into the pathophysiology of these disorders. This may also be relevant to non-pulmonary disorders that have been associated with low lung function, such as cardiovascular disorders [3,4] and diabetes [5], in which these genes may control systemic mechanisms (e.g. inflammation) that contribute to both low lung function and disease development.

Signal transducer and activator of transcription 3 (STAT3) is a member of a protein family of transcription factors, which was discovered in the course of studies of interferon-induced intracellular signal transduction [6]. These proteins are latent in the cytoplasm and become activated through tyrosine phosphorylation which typically occurs through cytokine receptor associated kinases (the Janus kinase-signal transducer or JAKs). The JAK-STAT pathway transmits information received from extracellular polypeptide signals, through membrane receptors, directly to target gene promoters in the nucleus, providing a mechanism for transcriptional regulation without second messengers. The gene, STAT3, is induced by a widearray of cytokines, including interleukin (IL)-6, IL-10, and IL-13, and has been implicated in the regulation of cell growth, inflammation, immune tolerance and early embryonic development. Recently, STAT3 was implicated in asthma pathogenesis in a study that showed that STAT3-dependent pathways induced by IL-13 in lung myofibroblasts were inhibited by the administration of the inhaled corticosteroid, fluticasone [7]. This suggests a role in airway inflammation and remodeling in asthmatics, which may affect lung function level.

In a previous study of the pharmacogenetics of asthma treatment, STAT3 was one of the candidate genes that we screened for association with response to cortocosteroid treatment [8]. Single nucleotide polymorphisms (SNPs) in STAT3 were genotyped and tested in a screening dataset from an adult asthma clinical trial. No effect of STAT3 SNPs on asthma drug response was seen in that study. However, the polymorphisms affected baseline lung function. In this report, we present our analysis of the association of STAT3 SNPs with lung function in adults with asthma, and replicate our findings in a cohort of children with asthma.

\section{Methods \\ Populations and Study Samples}

We used information from two asthma clinical trials, as previously reported [8]. All patients or their legal guardians consented to the study protocol and ancillary genetic testing. The Adult Study was a multicenter 8-week randomized clinical trial comparing the effect of once-daily high-dose inhaled flunisolide therapy with that of standard inhaled corticosteroid therapy (i.e. high vs standard dose inhaled corticosteroid therapy) among moderate to severe adult asthmatics [8]. Inclusion criteria were a history of asthma, $\geq 12 \%$ improvement in $\mathrm{FEV}_{1}$ with albuterol, and use of inhaled steroids at randomization. Exclusion criteria were non-asthma pulmonary disease, smoking ( $\geq 10$ pack-years), and recent asthma exacerbations requiring systemic steroids. Subjects were phoned weekly and had spirometry at 4 and 8 weeks. For this analysis, we included only the 401 Caucasian participants. The Childhood Asthma Management Program (CAMP) is a multicenter, randomized, double-blinded clinical trial testing the safety and efficacy of inhaled budesonide vs. nedocromil vs. placebo over a mean of 4.3 years. Trial design and methodology for CAMP have been published $[9,10]$. CAMP enrolled 1,041 children ages 5 to 12 years with mild to moderate asthma. Entry criteria included asthma symptoms and / or medication use for $\geq 6$-months in the previous year and airway responsiveness with a provocative concentration of methacholine causing a $20 \%$ reduction in $\mathrm{FEV}_{1}\left(\mathrm{PC}_{20}\right) \leq 12.5 \mathrm{mg} / \mathrm{ml}$. Data for 652 Caucasian children were included in this analysis.

\section{Phenotypes}

The primary phenotype of interest in both cohorts was baseline, pre-bronchodilator forced expiratory volume in one second, as a percentage of predicted $\left(\mathrm{PPFEV}_{1}\right)$. In the Adult Study, baseline $\mathrm{PPFEV}_{1}$ was measured after an openlabel 4-week period that demonstrated stability on the study drug or on standard inhaled therapy. Spirometry was then performed at monthly intervals, for a total of three spirometric measurements. In CAMP, baseline spirometry was performed at randomization, after a 28day period during which only as-needed albuterol was allowed. Follow-up spirometry was perfomed at 2, 4, 12, $16,24,28,36,40$, and 48 months. In addition to the analysis of the baseline $\operatorname{PPFEV}_{1}$, a repeated measures analysis was performed in both cohorts, making use of the longitudinal follow-up for each subject. In CAMP, we included information on parental smoking obtained from the baseline questionnaire.

\section{SNP Selection and Genotyping}

SNPs were selected from two sources, public databases and genomic DNA sequencing performed at the Whitehead Institute. Three SNPs were discovered as a result of the sequencing effort: G3363a3, G3363a4, and 
Table I: Flanking sequences for STAT3 SNPs

\begin{tabular}{lll}
\hline STAT3 & G3363al6 & GGGAAAATGAGATCAGGAGATAAAG [G/T]GGCACCCTTTGGTCTTGTAAAGCCTTTTTTA \\
STAT3 & G3363a3 & ACAGACATCATTTGAACTAGAGACTCT [G/A]TCTTTATTCAGAGATCTTCATTTTGTGGAC \\
STAT3 & G3363a4 & TCCCCTTCACAAAGGGCCTCTGGCTGC [C/G]GGAGAGGGCTAGGGAGAGCCTCACAG \\
STAT3 & rs 1026916 & AGGAAAAAGTTTAACCCAAAGACTGT [A/G]TGGATCTTCTCTACCCTACATCTCCAATCT \\
STAT3 & rs 1905340 & TATTTGAGAATCTAAGAAAGTAGATCA [A/C]ACTAAATATTGATATGCAGACACTAAAATC \\
STAT3 & rs 957971 & TGTTATATGAAGTGAATTAACCTCCTAT [C/G]GTACTTCAGTTTTCTCTATGCTAAAAGTGT \\
\hline
\end{tabular}

UCSC Genome Browser on Human May 2004 Assembly
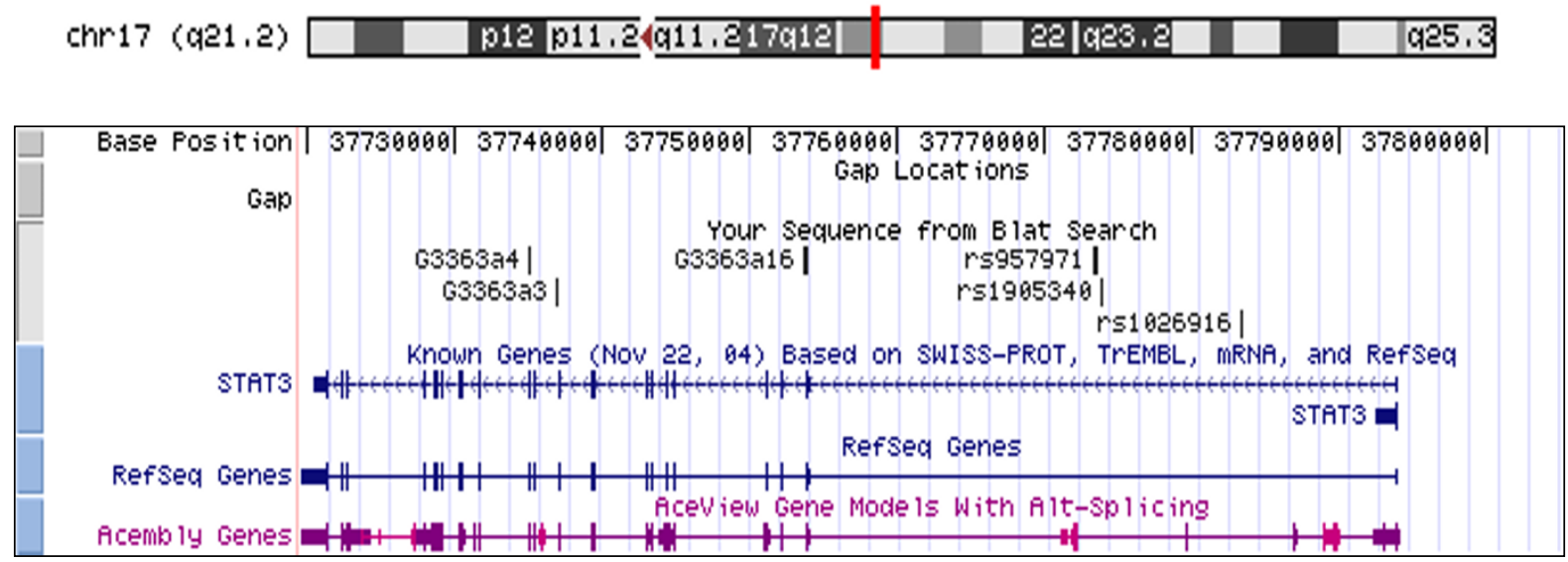

\section{Figure I}

Positions of the STAT3 SNPs genotyped in both the Adult Study and the CAMP cohort. From UCSC Genome Browser http://genome.ucsc.edu, May 2004 Human Reference Sequence Assembly[ I I, I 2]. UCSC webpage was accessed on March I8, 2005.

G3363a16. These three SNPs have been submitted to the public database and correspond precisely to rs8075442, rs2293152, and rs2306581, respectively (dbSNP: http:// www.ncbi.nlm.nih.gov/SNP). Five additional SNPs were chosen from public databases for genotyping, with the overall goal of having, on average, at least one SNP every 10 kilobases. Two of these SNPs - rs1803125 (exonic) and rs744284 (promoter) - were found to be monomorphic in the Adult Study subjects, and were not subsequently genotyped in the CAMP cohort. Three additional SNPs were successfully genotyped in both cohorts: rs1026916, rs1905340, and rs957971. These are all intronic SNPs (Figure 1[11,12]), and flanking sequences are given in Table 1. Linkage disequilibrium (LD) between each pair of SNPs was calculated and plotted using the LDPlotter tool http://innateimmunity.net/IIPGA2/Bioin formatics/, and expressed as the $\mathrm{r}^{2}$ LD statistic [13].
SNPs were genotyped via a SEQUENOM MassARRAY MALDI-TOF mass spectrometer (Sequenom, San Diego, CA) for analysis of unlabeled single-base extension minisequencing reactions with a semiautomated primer design program (SpectroDESIGNER, Sequenom). Our protocol implemented the very short extension method [14], whereby sequencing products are extended by only one base for three of the four nucleotides and by several additional bases for the fourth nucleotide (representing one of the alleles for a given SNP), permitting clearly delineated mass separation of the two allelic variants at a given locus.

\section{Statistical Analysis}

Single SNP association analyses were performed with SAS statistical software (SAS Institute, Inc., Cary, NC). Univariate associations between SNPs and the phenotype of 
interest $\left(\mathrm{PPFEV}_{1}\right)$ were tested by univariate linear regression, as implemented in Proc Reg in SAS. Multivariable linear regression models, were used to control for potential confounders. In these models, the genotype for each SNP was coded as three-level categorical variables (additive genetic model) or as dummy-coded variables. HardyWeinberg equilibrium for each SNP was tested using the chi-square goodness-of-fit test as implemented in the ALLELE Procedure in SAS.

The repeated measures analyses were carried out using a mixed linear model as implemented in the MIXED Procedure in SAS. A mixed linear model is a generalization of the standard linear model where the data are permitted to exhibit correlation and nonconstant variability, thereby providing the flexibility of modeling the variances and covariances of the data, in addition to the means. The covariance structure for the lung function data was specified using an unstructured model, which provided the best fit for the data after testing other covariance matrices (compound symmetry, spatial exponential, autoregressive, and autoregressive-heterogeneous). All models adjusted for time (in weeks) and contained a SNP $\times$ time interaction term. However, since the time interaction terms were not significant in any of the models, they were dropped from the final models presented in the results. Multiple testing for the single SNP association analyses was addressed by controlling the false discovery rate (FDR) using the method of Benjamini and Liu[15,16] (FDR tool available at https://innateimmunity.net/IIPGA2/Bioinformatics/. Control of the FDR was set at the 0.05 threshold.

Haplotype associations were explored with score tests that account for linkage phase ambiguity [17]. The score tests, derived from generalized linear models, are used for global tests of association, as well as haplotype-specific tests. The haplo.stats program implements the methods of Schaid et al, and was used for these analyses. Haplotypes were imputed and frequencies estimated using the modified EM algorithm estimation facility in haplo.stats. Analyses were run with and without adjustment for nongenetic factors. We modified the method to include data from individuals with partially missing marker information. The minimum haplotype frequency was set at $2.5 \%$. As previously reported [8] in an analysis of 59 SNPs across the genome, we found no evidence for population stratification in either population.

\section{Results}

Baseline characteristics and genotype frequencies of the six SNPs in both cohorts are shown in Table 2. G3363a3 in the Adult Study was the only SNP out of Hardy-Weinberg equilibrium, due to one rare individual who was homozygous for the minor allele. Genotype frequencies were also similar for both cohorts. Figure 2 plots the LD patterns among the six SNPs, with the corresponding $\mathrm{r}^{2}$ values in Table 3. The LD patterns are similar in the two cohorts.

Table 4 and Figure 3 show the results of linear regression models for baseline PPFEV ${ }_{1}$. In the Adult Study, three SNPs showed an effect on lung function. For G3363a16,

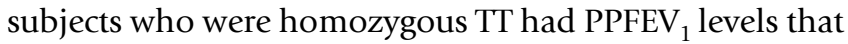
were $6.85 \%$ lower than levels for GG homozygotes. Likewise, for rs1026916 and rs957971, subjects who were homozygous for the minor allele had lower PPFEV values $_{1}$ than did subjects who were homozygous for the major allele. These results remained significant after controlling for the FDR (i.e. the p-values associated with each SNP were smaller than the FDR threshold p-value, thus we reject the null hypothesis of no significant association). In the CAMP trial, only rs1026916 was significantly associated with baseline $\operatorname{PPFEV}_{1}$, however, this did not remain statistically significant after controlling for the FDR (i.e. the p-value was greater than the FDR threshold p-value, thus we are unable to reject the null hypothesis of no significant association). Although not statistically significant, the direction of the changes in lung function associated with variation in G3363a16 and rs957971 paralleled those of the Adult Study. Similar results were obtained when we used raw pre-BD $\mathrm{FEV}_{1}$ measures, adjusted for age, sex, and height. Additional control for exposure to maternal smoking in utero or post-natal maternal or paternal smoking did not change the results. Interactions between individual SNPs and parental smoking variables were not significant. There was no significant association of any of the SNPs with forced vital capacity.

We then took the SNPs that were significant in the baseline analysis and performed a repeated measures analysis in each cohort, to take advantage of the multiple measures of $\mathrm{PPFEV}_{1}$. In the Adult Study, all 401 participants had complete data on $\mathrm{PPFEV}_{1}$ at all three time points. In the repeated measures analysis, results were similar to those of the analyses on baseline PPFEV $_{1}$ (Table 5), although only the results for G3363a16 and rs957971 remained significant after control for FDR. Since there were no differences in $\mathrm{PPFEV}_{1}$ levels at baseline or at any time point between the two treatment groups, we did not additionally control for treatment group in the multivariable longitudinal models. For CAMP, 6358 observations were available for analysis: 557 (85.3\%) children had complete information for the 10 time points, 56 (8.6\%) had information for 9 time points, $18(2.8 \%)$ had information for 8 time points, and $22(3.4 \%)$ had information for 3 to 7 time points. In CAMP, rs 1026916 was associated with lung function over the 4 years of observation, and stratified analyses showed similar effects of the SNP across treatment groups. Although treatment group had a significant effect on $\mathrm{PPFEV}_{1}$ over time, a formal test for 
Table 2: Baseline characteristics and genotype frequencies for the 6 SNPs in both cohorts.

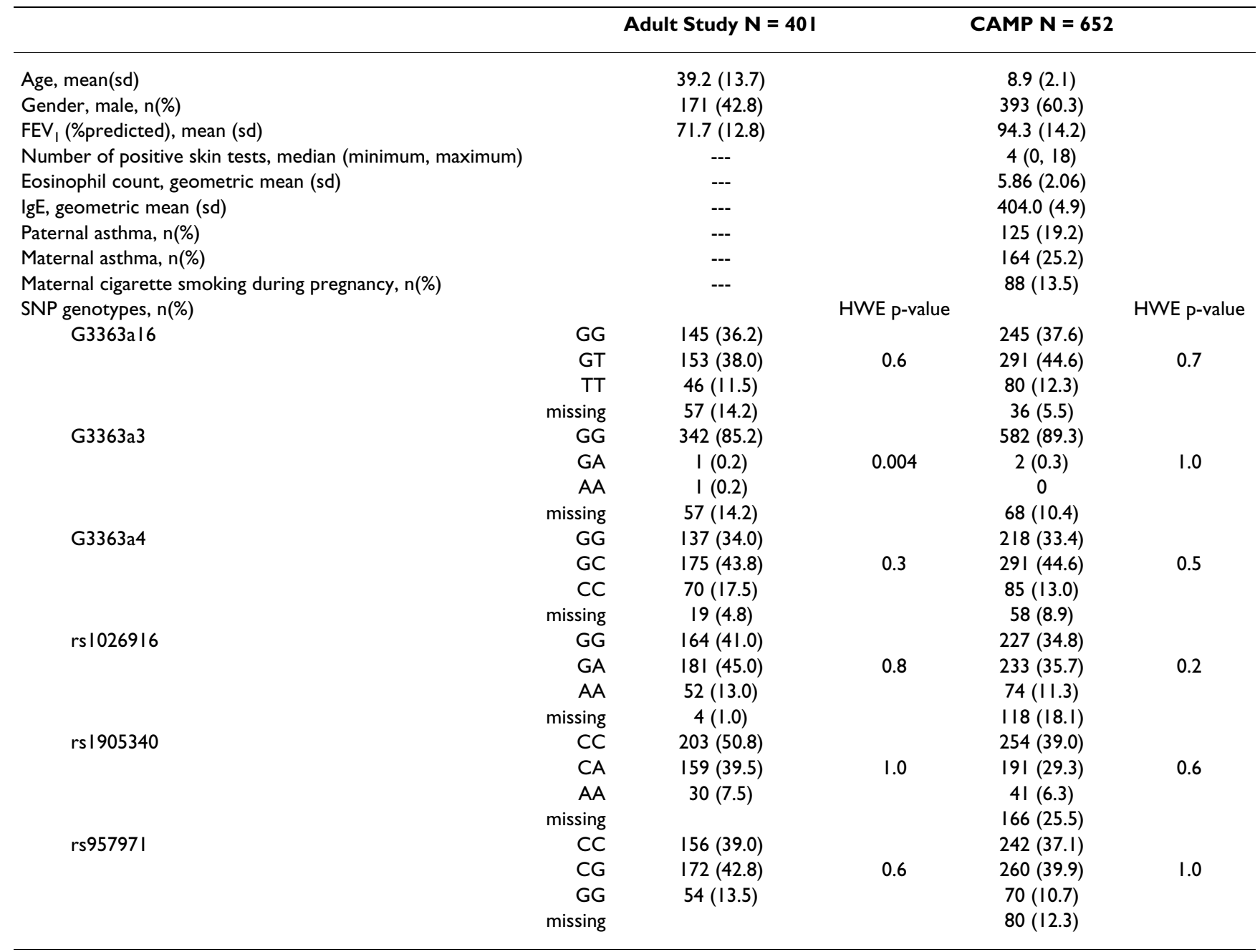

Table 3: Linkage disequilibrium $\left(r^{2}\right)$ among the six STAT3 SNPs*.

\begin{tabular}{lrrrrrr}
\hline $\begin{array}{l}\text { Adult Study } \\
\text { CAMP }\end{array}$ & G3363al6 & G3363a3 & G3363a4 & rs1026916 & rs1905340 & rs95797I \\
\hline G3363al6 & & 0.0089 & 0.1662 & 0.9804 & 0.6985 \\
G3363a3 & 0.0016 & & 0.0003 & 0.0076 & 0.0018 & 0.9936 \\
G3363a4 & 0.1668 & 0.0012 & 0.1418 & 0.1051 & 0.1666 \\
rs1026916 & 0.9754 & 0.0037 & 0.1627 & & 0.6693 \\
rs1905340 & 0.6563 & 0.0002 & 0.0888 & 0.6644 & 0.6718 \\
rs95797I & 0.9734 & 0.0036 & 0.1615 & 0.9854 & 0.6536 & \\
\hline
\end{tabular}

$*_{r} 2$ between SNPs for the Adult Study are above the diagonal and for the CAMP Study are below the diagonal

interaction between SNP and treatment group was not significant. Thus, treatment group was left as a covariate in the final models. In both cohorts, the SNP $\times$ time interac- tion terms were not significant, meaning that the effects of the individual SNPs did not vary over the time of each study. 

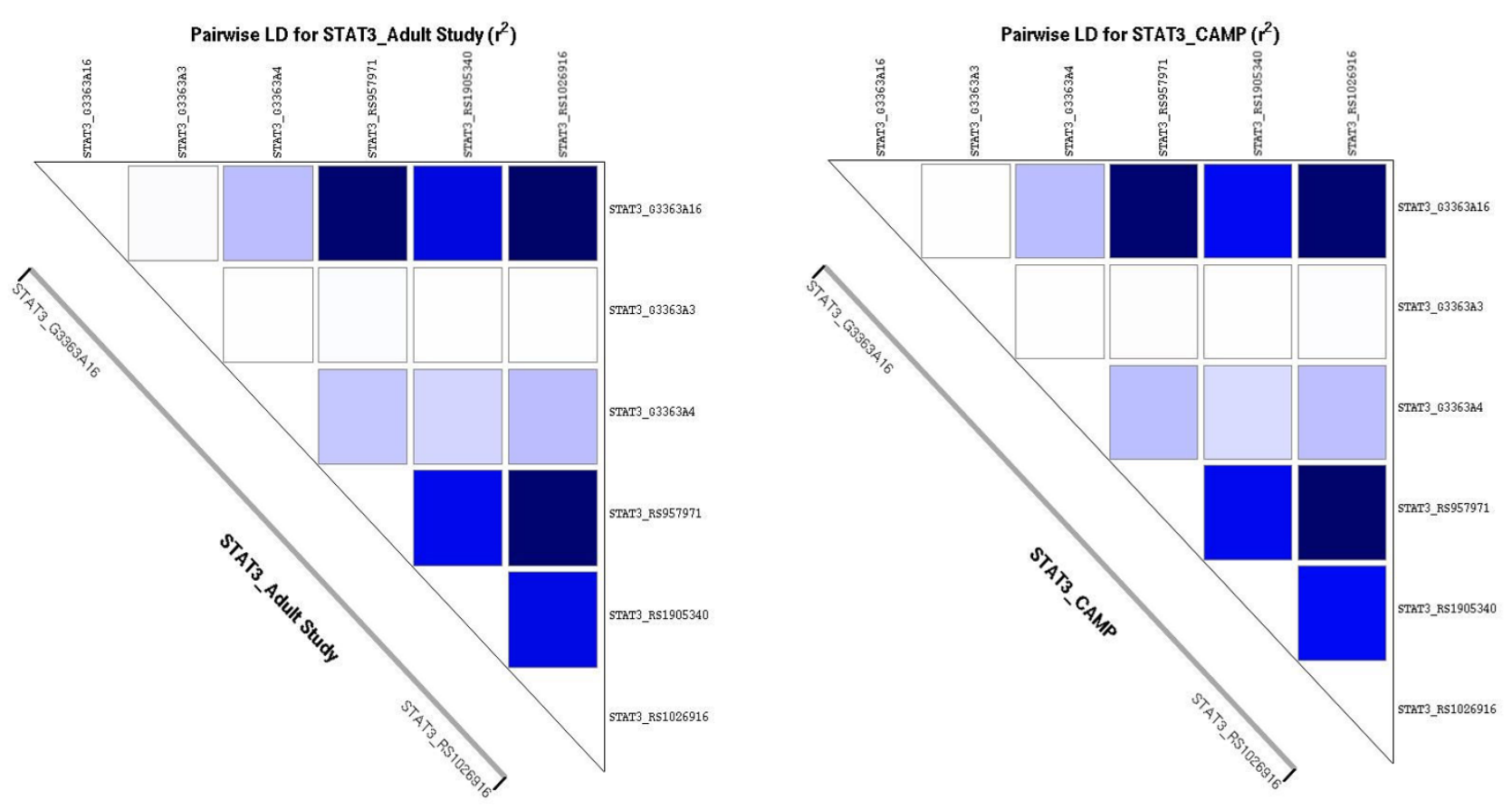

Figure 2

Linkage disequilibrium (LD) plots among the six STAT3 SNPs for both cohorts.LD is expressed as the $r^{2}$ statistic.

Haplotype analyses on baseline $\mathrm{PPFEV}_{1}$ were performed for each cohort. Table 6 presents the results for the Adult Study. There were five haplotypes that had frequencies above $2.5 \%$. The global statistic was significant at $\mathrm{p}=$ 0.02. Haplotype 5, which is comprised of the major alleles for G3363a16, rs957971, and rs1026916, was positively associated with $\operatorname{PPFEV}_{1}$, meaning that this haplotype was associated with higher PPFEV $_{1}$ values. On the other hand, haplotype 1, which contained the minor alleles of these three SNPs, was negatively associated with $\mathrm{PPFEV}_{1}$. These results were consistent with those of the single SNP analysis. Haplotype analysis in CAMP was also consistent with the single SNP analyses, but did not reach statistical significance (data not shown).

\section{Discussion}

This is the first report of an association between SNPs in the STAT3 gene and lung function in human populations. In this study, we show an association between SNPs in the STAT3 gene and FEV ${ }_{1}$ among asthmatics. These results are reasonably robust and are consistent in a cohort of adult asthmatics and a cohort of childhood asthmatics. Although only one SNP was significant in both cohorts, the direction of the effect of each individual SNPs was similar in the two cohorts. These effects were seen when we analyzed baseline $\mathrm{FEV}_{1}$ and the repeated measures of $\mathrm{FEV}_{1}$ over 8 weeks in the adult cohort and over 4 years in the childhood asthma cohort. In the cohort of childhood asthmatics, these effects were independent of parental smoking and asthma treatment group.

STAT proteins comprise a family of transcription factors latent in the cytoplasm, that are activated by a series of extracellular signaling proteins such as cytokine, growth factors, and hormones that bind to specific cell-surface receptors. The resulting signal transduction pathways permit them to play different roles in normal physiological cell processes, such as differentiation, proliferation, apoptosis, and angiogenesis [18,19]. Whereas other members of this gene family have generally demonstrated specificity in individual signaling pathways, STAT3 is deployed in various, sometimes disparate, physiological processes [6], including cell growth and differentiation [20], apoptosis [21], and anti-inflammatory processes mediated by IL-10 [22], to name a few. Additionally, while the different functions of the members of this gene family have been elucidated via targeted gene ablation, ablation of STAT3 leads to embryonic lethality in transgenic mice [23], underscoring its importance in embryogenesis. 
Table 4: Results of linear regression models for baseline PPFEV $_{1}{ }^{*}$

\begin{tabular}{|c|c|c|c|c|c|c|}
\hline \multirow[b]{2}{*}{ Stat3 SNPs } & \multicolumn{3}{|c|}{ Adult Study } & \multicolumn{3}{|c|}{ CAMP } \\
\hline & $\beta($ se) $\dagger$ & $\begin{array}{c}\text { Additive genetic } \\
\text { model p-value } \ddagger\end{array}$ & $\begin{array}{l}\text { FDR threshold } \\
\text { (decision§) }\end{array}$ & $\beta$ (se)† & $\begin{array}{l}\text { Additive genetic } \\
\text { model p-value } \ddagger\end{array}$ & $\begin{array}{l}\text { FDR threshold } \\
\text { (decision§) }\end{array}$ \\
\hline \multicolumn{7}{|l|}{ G3363al6 } \\
\hline Intercept (GG) & $74.03(1.06)$ & & & $95.67(0.92)$ & & \\
\hline GT & $-3.35(1.48)$ & 0.0007 & 0.007 & $-1.93(1.25)$ & 0.1 & 0.02 \\
\hline TT & $-6.85(2.15)$ & & (reject) & $-2.08(1.84)$ & & (accept) \\
\hline \multicolumn{7}{|l|}{ G3363a4 } \\
\hline Intercept (GG) & $70.76(1.10)$ & & & $94.19(0.98)$ & & \\
\hline GC & $1.18(1.47)$ & 0.3 & 0.05 & 1.06 (I.29) & 0.6 & 0.03 \\
\hline $\mathrm{CC}$ & $2.04(1.89)$ & & (accept) & $-2.04(1.86)$ & & (accept) \\
\hline \multicolumn{7}{|l|}{ rs1026916 } \\
\hline Intercept (GG) & $73.59(0.93)$ & & & $96.21(0.95)$ & & \\
\hline GA & $-2.90(1.38)$ & 0.004 & 0.01 & $-2.21(1.33)$ & 0.02 & 0.007 \\
\hline AA & $-5.22(2.03)$ & & (reject) & $-4.11(1.90)$ & & (accept) \\
\hline \multicolumn{7}{|l|}{ rs 1905340} \\
\hline Intercept (CC) & $73.04(0.89)$ & & & $95.52(0.89)$ & & \\
\hline$C A$ & $-3.00(1.34)$ & 0.04 & 0.03 & $-1.18(1.36)$ & 0.8 & 0.05 \\
\hline AA & $-2.77(2.49)$ & & (accept) & $0.70(2.39)$ & & (accept) \\
\hline \multicolumn{7}{|l|}{ rs95797I } \\
\hline Intercept (CC) & $73.48(1.02)$ & & & $95.56(0.01)$ & & \\
\hline CG & $-2.10(1.4 I)$ & 0.007 & 0.02 & $-2.07(1.26)$ & 0.08 & 0.01 \\
\hline GG & $-5.34(2.00)$ & & (reject) & $-2.64(1.91)$ & & (accept) \\
\hline
\end{tabular}

* Results are from individual linear regression models.

$\dagger \beta$ 's and $p$-values are from linear regression models with dummy-coded genotype categories.

$\neq \mathrm{p}$-values refer to the categorical genotype variable $(0,1,2)$, for the number of minor alleles present.

$\S$ Refers to the decision of whether to accept or reject the null hypothesis of no genotype effect. Please see text for further details.

In the lung, the function of STAT3 has not been fully elucidated. However, STAT3 appears to play a role in the regulation of surfactant [24,25], and in the inflammatory response in acute lung injury [26,27]. Additionally, STAT3 is an important mediator in the pro-inflammatory effects of the Th2 cytokine IL-13 on lung myofibroblasts $[7,28]$.

It is plausible that our results are due to an effect of the STAT3 gene during the embryonic stage of lung development. However, the results in the CAMP cohort show that this effect, if present, is likely a small one. On the other hand, STAT3 had stronger effects on lung function in the adult asthmatics, and a potential explanation for our findings is that STAT3 interacts with pro-inflammatory environmental stimuli, such as tobacco smoke, to affect FEV level. It is known that at least three factors determine lung function at a particular point in adult life: (1) the maximally attained level of lung function; (2) the onset of decline of lung function (or alternatively, the duration of the plateau phase); and (3) the rate of decline of lung function [29]. Whether STAT3 affects only a particular phase of lung growth or decline, or affects all phases remains to be seen. Furthermore, it needs to be deter- mined whether this effect of STAT3 on $\mathrm{FEV}_{1}$ level is unique to asthmatics or also applies to non-asthmatics.

Because the effects of STAT3 were stronger in the Adult Study, we additionally hypothesized that STAT3 may interact with environmental exposures to cause a decrement in lung function. Since cigarette smoking is established as the major environmental risk factor for low lung function[30], we hypothesized that exposure to cigarette smoke (either personal smoking or environmental tobacco smoke) could potentially interact with STAT3. We were unable to test the interaction between STAT3 and smoking in the Adult Study, because participants were non-smokers. In CAMP, we did not see a significant interaction effect between the individual SNPs and parental smoking variables (in utero smoke exposure, maternal smoking, paternal smoking), and too few children smoked to permit any meaningful interaction analyses. It is also possible that other environmental exposures that we did not measure could be interacting with STAT3.

A limitation of our study is the lack of complete sequence information on the gene. The sequencing efforts focused 




Figure 3

The association between STAT3 SNPs and FEV, . Mean ( \pm sd) percent predicted FEV in the Adult Study plotted against genotype for each SNP. Additive genetic models were statistically significant for each SNP: $p=0.0007,0.0043$, and 0.007 , respectively for G3363al6, rs1026916, and rs95797I.

Table 5: Effects of Stat3 SNPs on repeated measures of PPFEV, over time*

\begin{tabular}{|c|c|c|c|c|c|}
\hline \multirow[b]{2}{*}{ Stat3 SNPs } & \multicolumn{3}{|c|}{ Adult Study } & \multicolumn{2}{|c|}{ CAMP } \\
\hline & $\beta$ (se) & $\begin{array}{c}\text { Additive genetic } \\
\text { model p-value } \ddagger\end{array}$ & $\begin{array}{l}\text { FDR threshold } \\
\text { (decision§) }\end{array}$ & $\beta$ (se) & $\begin{array}{l}\text { Additive genetic } \\
\text { model p-value }\end{array}$ \\
\hline \multicolumn{6}{|l|}{ G3363al6 } \\
\hline Intercept (GG) & $73.85(1.21)$ & & & & \\
\hline GT & $-2.72(1.69)$ & 0.008 & 0.009 & --- & --- \\
\hline TT & $-6.33(2.46)$ & & (reject) & & \\
\hline \multicolumn{6}{|l|}{ rs1026916 } \\
\hline Intercept (GG) & $73.46(1.13)$ & & & 97.01 (0.93) & \\
\hline GA & $-2.25(1.56)$ & 0.028 & 0.017 & $-0.19(0.99)$ & 0.02 \\
\hline$A A$ & $-4.74(2.30)$ & & (accept) & $-4.24(1.49)$ & \\
\hline \multicolumn{6}{|l|}{ rs95797I } \\
\hline Intercept (CC) & $73.36(1.16)$ & & & & \\
\hline CG & $-1.51(1.60)$ & 0.036 & 0.038 & --- & --- \\
\hline GG & $-5.04(2.29)$ & & (reject) & & \\
\hline
\end{tabular}

* $\beta$ 's obtained from mixed models of repeated measurements of percent predicted FEV, over time. All models are adjusted for time in weeks, with a maximum of 3 observations for the Adult Study, and 10 observations for the CAMP study. The model for CAMP is adjusted additionally for treatment group.

§Refers to the decision of whether to accept or reject the null hypothesis of no genotype effect. Please see text for further details. 
Table 6: Association of haplotypes in the STAT3 gene and baseline PPFEV in the Adult Study.

\begin{tabular}{|c|c|c|c|c|c|c|c|c|c|}
\hline \multicolumn{7}{|c|}{ Haplotypes } & \multirow{2}{*}{ Frequency } & \multirow{2}{*}{$\begin{array}{c}\text { Haplotype } \\
\text {-Specific } \\
\text { Score } \\
\text { Statistic }\end{array}$} & \multirow[t]{2}{*}{ p-value } \\
\hline & G3363a4 & G3363a3 & G3363al6 & rs957971 & rs 1905340 & rs 1026916 & & & \\
\hline (I) & $\bar{C}$ & $\overline{\mathrm{G}}$ & $\bar{T}$ & $\overline{\mathrm{G}}$ & $\bar{A}$ & $\bar{A}$ & 0.04501 & -1.91 & 0.05 \\
\hline (2) & G & G & $\mathrm{T}$ & G & A & A & $0.2344 I$ & -1.73 & 0.08 \\
\hline (3) & G & G & $\mathrm{T}$ & G & C & $A$ & 0.06895 & -1.60 & 0.1 \\
\hline (4) & G & G & G & C & $\mathrm{C}$ & G & 0.27914 & 0.96 & 0.3 \\
\hline (5) & C & G & G & C & C & G & 0.35557 & 2.58 & 0.01 \\
\hline
\end{tabular}

Global Score Statistics:

Global Statistic: 13.3, df $=5$

Global p-value $=0.02$

only on the exons, thus limiting our knowledge of the full linkage disequilibrium pattern of the gene. Furthermore, since all the SNPs we successfully genotyped were in intronic regions of the gene, it is likely that these are not responsible for the association but are rather in linkage disequilibrium with the functional variant of this gene, or with variants in another gene. Studying other populations, such as general population samples or occupational cohorts with exposure information, may help elucidate the effects of this gene on $\mathrm{FEV}_{1}$. Additionally, more functional studies of SNPs in STAT3 are needed to elucidate its role in lung function development.

Airway hyperresponsiveness was not assessed in the Adult Study. However, the subjects had a physician diagnosis of moderate to severe asthma (as evidenced by the levels of lung function), were on inhaled steroids at baseline, and had a significant bronchodilator response to albuterol. The combination of a physician diagnosis and bronchodilator response is a reasonable definition of asthma in genetic studies[31]. Furthermore, significant smoking and non-asthma respiratory disorders were excluded. Additionally, we did not have information on allergy outcomes in the Adult Study, either. In CAMP, we performed additional analyses, however, there were no associations between any of the STAT3 SNPs or either serum IgE level or skin test reactivity.

We controlled for multiple testing by controlling the false discovery rate. An additional strategy we took to minimize the effect of multiple testing is by performing a screening analysis in the Adult Study, then performing a replicate analysis in CAMP. Population stratification is another potential concern [32]; thus, we included only Caucasian subjects in this analysis. Furthermore, in previous testing utilizing a panel of 59 random markers, we found no evidence for stratification in the Caucasian subjects in either of these two cohorts [8].

\section{Conclusion}

We have shown that polymorphisms in STAT3 are associated with $\mathrm{FEV}_{1}$ in asthmatics. We show these effects both in a cohort of adult asthmatics and in a cohort of childhood asthmatics. In both cohorts, we excluded gross population stratification by testing with a panel of random markers. The precise mechanism for the effects of this gene on $\mathrm{FEV}_{1}$ remains unknown. However, while we see an association between SNPs in this gene and $\mathrm{FEV}_{1}$ in young asthmatics, the effects were stronger in the adult asthmatics, suggesting a role of STAT3 in chronic inflammatory pathways that may have an impact on lung growth and decline.

\section{Authors' contributions}

AAL participated in the conceptualization of the analysis, designed the analysis, performed the analyses and interpretation of results, and drafted the manuscript. KGT participated in the selection and genotyping of SNPs, conception and design of the study, acquisition of the data, and drafting and critically revising the manuscript. SL participated in the statistical analysis of the data and in critically revising the manuscript. RL participated in the conception and design of the analysis, and in critically revising the manuscript. BGR participated in the preparation of the data for analysis and in critically revising the manuscript. SG participated in the selection and genotyping of SNPs and in critically revising the manuscript. ESS participated in the design of the analysis and in critically revising the manuscript. STW conceived of the study, participated in its design and its coordination, participated in acquisition of the data, and participated in critically revising the manuscript.

\section{Grant Support}

This work was supported by: U01 HL065899 - The Pharmacogenetics of Asthma Treatment; the Childhood Asthma Management Program (CAMP) by contracts N01-HR- 
16044, 16045, 16046, 16047, 16048, 16049, 16050, 16051, and 16052; and the CAMP Genetics Ancillary Study by P01 HL067664, all awarded by the NHLBI.

\section{Acknowledgements}

The authors wish to acknowledge Dr. David Altshuler and Dr. Eric Lander for their assistance with the generation of sequence data utilized in the genotyping assays. We thank all the CAMP families for their enthusiastic participation in the CAMP Genetics Ancillary Study. We also acknowledge the Childhood Asthma Management Program (CAMP) investigators and research team, supported by the National Heart, Lung, and Blood Institute (NHLBI) at the National Institutes of Health (NIH), Bethesda, Maryland USA, for collection of CAMP Genetics Ancillary Study data. All work on data from the CAMP Genetics Ancillary Study was conducted at the Channing Laboratory, Brigham and Women's Hospital under appropriate CAMP policies and human subjects protections.

\section{References}

I. Rybicki BA, Beaty TH, Cohen BH: Major genetic mechanisms in pulmonary function. J Clin Epidemiol 1990, 43:667-675.

2. Givelber RJ, Couropmitree NN, Gottlief DJ: Segregation analysis of pulmonary function among families in the Framingham Study. Am J Respir Crit Care Med 1998, 157:|445-145I.

3. Friedman GD, Klatsky AL, Siegelaub M: Lung function and risk of myocardial infarction and sudden cardiac death. $N$ EnglJ Med 1976, 294: 107|-1075.

4. Lange P, Nyboe J, Jensen G, Schnohr P, Appleyard M: Ventilatory function impairment and risk of cardiovascular death and of fatal or non-fatal myocardial infarction. Eur Respir J I99I, 4:1080-1087.

5. Walter RE, Beiser A, Givelber RJ: Association between glycemic state and lung function: The Framingham Heart Study. $\mathrm{Am} J$ Resp Crit Care Med 2003, 167:911-916.

6. Levy DE, Lee C: What does Stat3 do? J Clin Invest 2002, 109: I I43-1148.

7. Cazes E, Giron-Michel J, Baouz S, Doucet C, Cagnoni F, Oddera S, Korner M, Dasic G, Testi R, Azzarone B, Canonica GW: Novel antiinflammatory effects of the inhaled corticosteroid fluticasone propionate during lung myofibroblastic differentiation. J Immunol 200I, 167:5329-5337.

8. Tantisira KG, Lake S, Silverman ES, Palmer LJ, Lazarus R, Silverman EK, Liggett SB, Gelfand EW, Richter B, Israel E, Gabriel S, Altshuler D, Lander E, Drazen J, Weiss ST: Corticosteroid Pharmacogenetics: Association of sequence variants in CRHRI with improved lung function in asthmatics treated with inhaled corticosteroids. Hum Mol Genet 2004, I3:1353-1359.

9. The Childhood Asthma Management Program (CAMP); design, rationale, and methods. Childhood Asthma Management Program Research Group. Control Clin Trials 1999, 20:91-120.

10. Long-term effects of budesonide or nedocromil in children with asthma. Childhood Asthma Management Program Research Group. N Engl J Med 2000, 343:1054- 1063.

II. Kent WJ, Sugnet CW, Furey TS, Roskin KM, Pringle TH, Zahler AM, Haussler D: The human genome browser at UCSC. Genome Res 2002, I 2:996-1006.

12. Karolchik D, Baertsch R, Diekhans M, Furey TS, Hinrichs A, Lu YT, Roskin KM, Schwartz M, Sugnet CW, Thomas DJ, Weber RJ, Haussler $D$, Kent W]: The UCSC genome browser database. Nucl Acids Res 2003, 31:5I-54.

13. Carlson CS, Eberle MA, Rieder MJ, Yi Q, Kruglyak L, Nickerson DA: Selecting a maximally informative set of single-nucleotide polymorphisms for association analyses using linkage disequilibrium. Am J Hum Genet 2004, 74:106-120.

14. Sun X, Ding H, Hung K, Guo B: A new MALDI-TOF based minisequencing assay for genotyping of SNPS. Nucleic Acids Res 2000, 28:E68.

15. Benjamini $Y$, Liu W: A distribution-free multiple test procedure that controls the false discovery rate. Tel Aviv, Tel Aviv University; 1999.
16. Benjamini Y, Drai D, Elmer G, Kafkafi N, Golani I: Controlling the false discovery rate in behavior genetics research. Behav Brain Res 200I, I 25::279-284.

17. Schaid DJ, Rowland CM, Tines DF, Jacobson RM: Score tests for association between traits and haplotypes when linkage phase is ambiguous. Am J Hum Genet 2002, 70:425-434.

18. Horvath C: STAT proteins and transcriptional responses to extracellular signals. Trends Biochem Sci 2000, 25:496-502.

19. Calo V, Migliavacca M, Bazan V, Macaluso M, Buscemi M, Gebbia N, Russo A: STAT proteins: from normal control of cellular events to tumorigenesis. J Cell Physiol 2003, 197:157-168.

20. Bromberg JF, Wrzeszczynska MH, Devgan G, Zhao Y, Pestell RG, Albanese C, Darnell JEJ: Stat3 as an oncogene. Cell 1999, 98:295-303.

21. Chapman RS, Lourenco P, Tonner E, Flint D, Selbert S, Takeda K, Akira $S$, Clarke AR, Watson CJ: The role of Stat3 in apoptosis and mammary gland involution. Conditional deletion of Stat3. Adv Exp Med Biol 2000, 480:129-138.

22. Williams L, Bradley L, Smith A, Foxwell B: Signal transducer and activator of transcription 3 is the dominant mediator of the anti-inflammatory effects of IL- 10 in human macrophages. J Immunol 2004, I72:567-576.

23. Takeda K, Noguchi K, Shi W, Tanaka T, Matsumoto M, Yoshida N, Kishimoto T, Akira S: Targeted disruption of the mouse Stat3 gene leads to early embryonic lethality. Proc Natl Acad Sci U S A 1997, 94:380I-3804.

24. Yan C, Naltner A, Martin M, Naltner M, Fangman JM, Gurel O: Transcriptional stimulation of the surfactant protein $B$ gene by STAT3 in respiratory epithelial cells. J Biol Chem 2002, 277: 10967-10972.

25. Yang L, Lian X, Cowen A, Xu H, Du H, Yan C: Synergy between Stat 3 and RAR\{alpha\} in Regulation of the Surfactant Protein B Gene in the Lung. Mol Endocrinol 2004, 18:1520-1532.

26. Severgnini M, Takahashi S, Rozo LM, Homer RJ, Kuhn C, Jhung JW, Perides G, Steer M, Hassoun PM, Fanburg BL, Cochran BH, Simon AR: Activation of the STAT Pathway in Acute Lung Injury. Am J Physiol Lung Cell Mol Physiol 2004, 286:LI 282-92.

27. Hokuto I, Ikegami M, Yoshida M, Takeda K, Akira S, Perl AK, Hull WM, Wert SE, Whitsett JA: Stat-3 is required for pulmonary homeostasis during hyperoxia. J Clin Invest 2004, I 1 3:28-37.

28. Doucet C, Giron-Michel J, Canonica GW, Azzarone B: Human lung myofibroblasts as effectors of the inflammatory process: the common receptor gamma chain is induced by Th2 cytokines, and CD40 ligand is induced by lipopolysaccharide, thrombin and TNF-alpha. Eur I Immunol 2002, 32:2437-2449.

29. Weiss ST, Ware JH: Overview of issues in the longitudinal analysis of respiratory data. Am J Respir Crit Care Med 1995, 154:S208-S2II.

30. Sherrill DL, Lebowitz MD, Burrows B: Epidemiology of chronic obstructive pulmonary disease. Clin Chest Med 1990, I I:375-388

3I. Celedon JC, Silverman EK, Weiss ST, Wang B, Fang Z, Xu X: Application of an algorithm for the diagnosis of asthma in Chinese families: limitations and alternatives for the phenotypic assessment of asthma in family-based genetic studies. $\mathrm{Am} J$ Resp Crit Care Med 2000, 162:1679-1684.

32. Silverman EK, Palmer L): Case-control association studies for the genetics of complex respiratory diseases. Am J Respir Cell Mol Biol 2000, 22:645-648. 